\title{
TAP1 Gene Polymorphism in Alopecia Areata
}

E.M.Sanad ${ }^{1}$, R.M.Salem ${ }^{1}$, H.M.Abdel Kareem ${ }^{2}$ and SH.S.Abdelhamid ${ }^{1}$

${ }^{1}$ Dermatology and Andrology Dept., Faculty of Medicine, Benha Univ., Benha, Egypt

${ }^{2}$ Medical Biochemistry Dept., Faculty of Medicine, Benha Univ., Benha, Egypt

E-Mail: shemoo62010@gmail.com

\begin{abstract}
Alopecia areata (aa) is a common type of hair loss. It is an autoimmune disease with a variable, typically relapsing or remitting, course that can be persistent-especially when hair loss is extensive. Three major phenotypic variants of the disease are patchy-type aa, alopecia universalis and alopecia totalis. The aim of the present study was to assess the relation between TAP1(rs1057141) gene polymorphism and alopecia areata risk and its clinical criteria. This study was carried out on 100 patients suffering from Alopecia Areata and 100 apparently healthy individuals of matched age and sex as a control group. Patients were recruited from the outpatient clinic of Dermatology and Andrology Department of Benha University Hospitals during the period from August 2018 to January 2019. There was insignificant difference in TAP1 (rs1057141) genotypes in both studied groups. TAP1 (rs1057141) gene polymorphism does not have a role in alopecia areata development.
\end{abstract}

Keywords: Alopecia areata, Gene polymorphism, TAP1.

\section{Introduction}

Alopecia areata (aa) is a common type of hair loss; it is an autoimmune disease with a variable, typically relapsing or remitting, course that can be persistent-especially when hair loss is extensive. Alopecia areata is the second most frequent nonscarring alopecia, after male and female pattern alopecia [1].

The three major phenotypic variants of the disease are patchy-type aa, which is often localized to small ovoid areas on the scalp or in the beard area, alopecia totalis, which involves the entire scalp, and alopecia universalis, which involves the entire body surface area [2].

The transport TAP-1 binding cassette sub-family $\mathrm{B}$ gene (TAP1) has been localized to the major histocompatibility comlex (MHC) class II region. The protein forms aheterodiemer with TAP2, which transports antigenic peptides from the cytosol into the endoplasmic reticulum lumen, prior to assembly of class I molecules [3].

It has been shown that polymorphisms in TAP1 or TAP2 may affect antigen recognition and presentation, potentially resulting in low or entirely absent cell surface expression of MHC-I molecules, and an adverse immune response [4].

\section{Aim of work}

The aim of this study was to assess the relation between TAP1 (rs1057141) gene polymorphisms and alopecia areata risk and its clinical criteria.

\section{Subjects and methods}

Type of the study is a case control study. This study was carried out on 100 patients suffering from alopecia areata and 100 apparently healthy individuals of matched age and sex as a control group. Patients were recruited from the outpatient clinic of Dermatology and Andrology Department of Benha University Hospitals during the period from August 2018 to January 2019.

\subsection{Ethical considerations}

The study was approved by the local ethics committee on research involving human subjects of Benha Faculty of Medicine. Informed consent was obtained from each individual before sample collection.

\subsection{Exclusion criteria}

Any subject was excluded from the study if he/ she were suffering from any of the following conditions: Inflammatory, autoimmune or infectious cutaneous or systemic diseases, chronic systemic illness e.g. hepatic, renal, cardiac diseases and associated malignancy. Pregnancy or lactation were also exclusion criteria.

\subsection{Methods}

All patients were subjected to the followings: 3.3.1 Full History Taking

Personal history: Name, age, sex, special habits of medical importance, alopecia, site, stress as provacating factor, family history of alopecia, history of previous treatment and response to it and history of eye, ear, and nail affection.

\subsubsection{Dermatological examination}

Description of the alopecia lesions including site, type and number of patches. Nail examination to detect any associated nail disease. Disease severity in alopecia areata patients was assessed by (SALT score).

\subsubsection{Laboratory investigations}

All studied subjects were tested for TAP1 (rs1057141) gene polymorphism using real time PCR technique.

\subsection{Sampling}

Three ml venous blood was collected from each subject by clean venipuncture using disposable 
plastic syringe and placed on ethylene diamine tetraacetic salt (EDTA) $(1.2 \mathrm{mg} / \mathrm{mL})$ as an anticoagulant and stored at $80^{\circ} \mathrm{C}$ for molecular assay of TAP 1 genotypes.

\section{DNA extraction}

DNA extraction from whole blood by GENE JET Genomic DNA Purification Kits (Qiagen) was done according to manufacture instructions.

\subsection{Statistical methods}

The collected data was revised, coded and tabulated using Statistical package for Social Science (IBM Corp. Released 2011. IBM SPSS Statistics for Windows, Version 20.0. Armonk, NY: IBM Corp.). Data were presented and suitable analysis was done according to the type of data obtained for each parameter.

\section{Result and discussion \\ 4.1 Result \\ Demographic data}

There was non-significant difference between cases and control groups regarding age and sex.

Gene Study:

The studied SNP are located on the short arm of chromosome 6 with TAP1 gene. Features of studied SNP are displaced in Table (1). Both patients and control subjects groups were in Hardy Weinberg equilibrium, no significant difference were found between them Table (2).

TAP1 genotypes and alleles in the studied SNP:

There was insignificant difference in SNP (rs1057141) genotypes distribution between patient and control groups Table (3).

Table (1) Gene study.

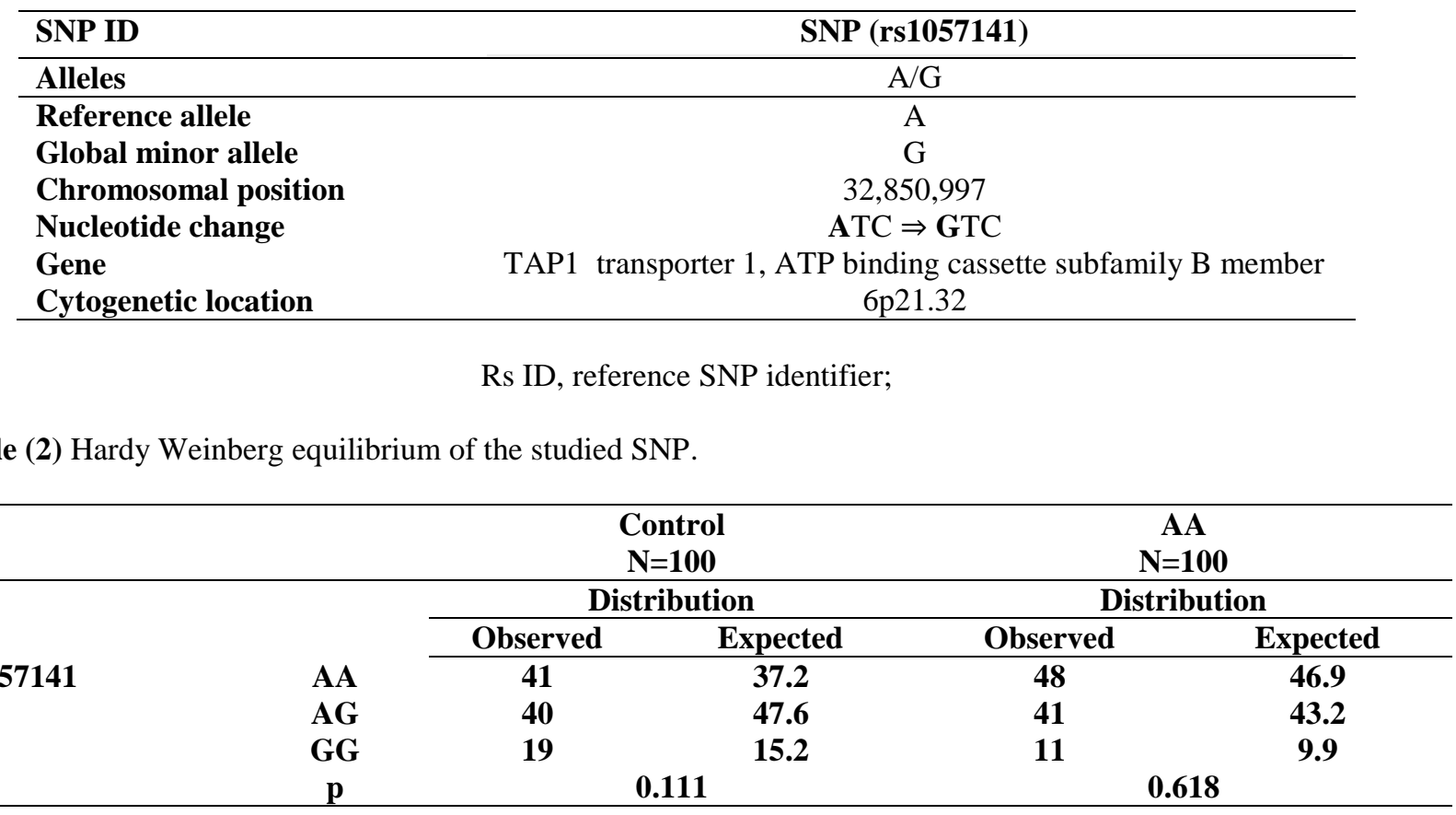

HW, Hardy Weinberg. Deviations from Hardy-Weinberg equilibrium expectations were determined using the chisquared test.

Table (3)TAP1 genotypes and alleles in both groups.

\begin{tabular}{|c|c|c|c|c|c|c|c|c|}
\hline & \multicolumn{2}{|c|}{$\begin{array}{l}\text { Control } \\
\mathrm{N}=100\end{array}$} & \multicolumn{2}{|c|}{$\begin{array}{l}\text { AA } \\
N=100\end{array}$} & \multirow[t]{2}{*}{$\mathbf{p}$} & \multirow[t]{2}{*}{ OR } & \multirow[t]{2}{*}{$95 \%$ CI } \\
\hline & & $\mathbf{N}$ & $\%$ & $\mathbf{N}$ & $\%$ & & & \\
\hline \multirow[t]{6}{*}{ rs1057141 } & $\mathbf{A A}$ & 41 & 41 & 48 & 48 & & 1 & (reference) \\
\hline & AG & 40 & 40 & 41 & 41 & 0.666 & 0.920 & $0.631-1.342$ \\
\hline & GG & 19 & 19 & 11 & 11 & 0.102 & 0.644 & $0.380-1.092$ \\
\hline & AG+GG & 59 & 59 & 52 & 52 & 0.319 & 0.837 & $0.590-1.188$ \\
\hline & $\mathbf{A}$ & 122 & 61.0 & 137 & 68.5 & & 1 & (reference) \\
\hline & G & 78 & 39.0 & 63 & 31.5 & 0.116 & 0.719 & $0.476-1.086$ \\
\hline
\end{tabular}

4.2 Discussion 
Alopecia areata is a common, inflammatory, nonscarring type of hair loss. Significant variations in the clinical presentation of aa have been observed, ranging from small, well-circumscribed patches of hair loss to a complete absence of body and scalp hair [5].

Alopecia areata is an autoimmune condition that is characterized by non-scarring hair loss. Its aesthetic repercussions can lead to profound changes in the psychological well-being [6].

The aim of this study was to assess the relation between TAP1 gene polymorphisms and alopecia areata risk and its clinical criteria.

According to the National Center for Biotechnology Information, the genetic features of the studied SNP reported that (rs1057141) is located on the short arm of chromosome 6 within TAP1 gene, and had $A$ and $G$ alleles. $A$ is the reference, and $G$ is the minor allele [7].

Regarding the studied SNP (rs1057141), there was no significant difference between patient and control groups in the genotypes distribution. The (rs1057141) genotypes distribution was not related to any of the studied variables. This comes in harmony with Kim et al. [8] who studied the relation between the same SNP and aa in a sample of Korean population.

Alopecia areata has many associations. Atopy is one of the most common associations with aa [9]. The TAP1 (rs1057141) was reported to be associated with increased risk of atopic disease [10] Among the common association with alopecia areata is other autoimmune diseases such as diabetes type1, and autoimmune anemia [11] autoimmune thyroiditis [12] Absence of significant difference between the TAP1 polymorphism between the currant patients and control subjects regarding the first SNP, while its relation to other disease known to be associated with alopecia areata may support the multifactorial nature of aa. Moreover, this confirms the role of other environmental factors.

Stress has a very importance role in aa development through cytokines release which regulate innate and adaptive immune responses. These include tumor necrosis factor alpha (TNF $\alpha)$ or interferon gamma (IFY). They participate in cellular adaptive immune responses traditionally addressed as T-helper cell type1 (TH1). In response to prolonged stress exposure, the epithelial and mesenchymal cells in the skin are driven into apoptosis and premature termination of hair growth occurs [13]

Alfani et al. [14] reported that patients with alopecia areata appeared to experience more depressive, hysterical and anxiety feelings (stressful events), as aa usually impact their social relationships.

[12] S.Lee, Y.B.Lee, Screening of thyroid function and autoantibodies in patients with alopecia areata, systematic review and meta-

\section{Conclusion}

TAP1 (rs1057141) gene polymorphism does not have a role in alopecia areata development.

\section{References}

[1 ] C.H.Pratt, L.E.King J.r, A.G.Messenger, A.M.Christiano, and J.P.Sundberg, Alopeciaareata Nature reviews Disease primers, Vol.3, PP. 17011, 2017.

[2] A.Christiano, and R.Clynes, Epidemiology of alopecia areata: systematic review. U.S. Patent Application, Vol.15152-205, 2019.

[3 ] Y.Miao, Z.Kang, F.Xu and S.Qi, Association analysis of the IL2RA gene with alopecia areata in Chinese population.Dermatology, Vol. 227, PP.229-304, 2013.

[4 ] D.Wang, Y.Zhou, L.Ji, T.He, F.Lin, R.Lin, Association of LMP/TAP gene polymorphisms with tuberculosis susceptibility in Li population in China.PLoS One, vpl.7(3), PP. e33051, 2012.

[5 ] L.C.Strazzulla, E.H.C.Wang, L.Avila, K.L.Sicco, N.Brinster, A.M.Christiano, and J .Shapiro, Alopecia areata: Disease characteristics, clinical evaluation, and new perspectives on pathogenesis, Journal of the American Academy of Dermatology, Vol.78(1), PP. 1-12, 2018.

[6 ] J.Rajoo, G.Wong, I.S.Cooper, D.J.Raj, A.H.Castle J.Chong, G.A.Green, KennedyAlopecia areata and physosis. BMC psychology, Vol.7(1), PP. 48, 2019.

[7 ] E.Bernardis, and L.Castelo-Soccio, Quantifying Alopecia Areata via Texture Analysis to Automate the SALT Score Computation, Journal of Investigative Dermatology, Vol.19, PP.S34-S40, 2018.

[8 ] H.K.Kim, H.Lee, B.L.Lew, W.Y.Sim, Y.O.Kim, S.W.Lee and H.J.Kim, Association between TAP1 gene polymorphisms and alopecia areata in a Korean population, Genet Mol Res, Vol.14(4), PP. 18820-7, 2015.

[9 ] N.Aszoide, T.Pumena, A.Wollenberg, Dupilumab associated healing of alopecia areata in atopic dermatitis patient. Deutsche Medizinische Wochenschrift,vol.144(9), PP. 602-605, 2019.

[10 ] S.Stemmler, S.Hoffjan, Trying to understand the genetics of atopic dermatitis.Mol Cell Probes, Vol.30, PP.374-85, 2016.

[11 ] N.Islam, P.S.Leung and A.C.Huntley, The autoimmune basis of alopecia areata. A comprehensive review. Autoimmune Rev, Vol.14(2), PP. 81-90, 2015.

analysis. J Am Acad Dermatol, Vol.80, PP.14103, 2019. 
[13 ] F.E.Huang, R.C. Rodríguez, J.T.Brandenburg, F.Carpentier, and T.Giraud, Gene Presence-Absence Polymorphism in Castrating Anther-Smut Fungi, Recent Gene Gains and Phylogeographic Structure. Genome biology and evolution, Vol.10(5), PP. 12981314, 2017.
[14] S.lfani, V.Antinone, A.Mozzetta, C.D.Pietro, C.azzanti, P.Stella, and D.beni, Psychological status of patients with alopecia areata. Actadermato-venereologica, Vol.92(3), pp.304-306, 2012. 